\title{
Colloidal dynamics and elasticity of dense wax particle suspensions over a wide range of volume fractions when tuning the softness by temperature
}

\author{
Jiwon Yoon ${ }^{\mathrm{a}}$, Frank Scheffold ${ }^{\mathrm{b}}$, Kyung Hyun Ahn ${ }^{\mathrm{a}, *}$ \\ ${ }^{a}$ School of Chemical and Biological Engineering, Seoul National University, 599 Kwanak-ro Kwanak-gu, South Korea \\ ${ }^{\mathrm{b}}$ Department of Physics, University of Fribourg, Chemin du Muse 3, 1700 Fribourg, Switzerland
}

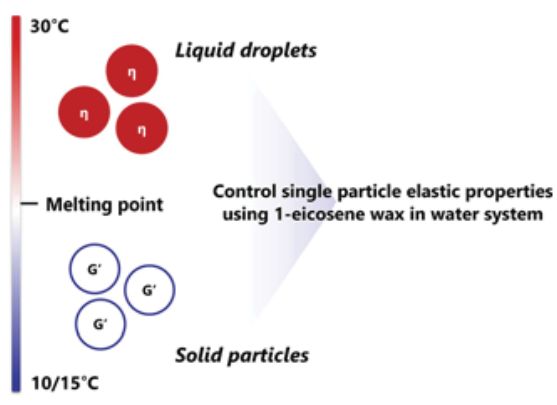

\section{A R T I C L E IN F O}

\section{Keywords:}

Wax

Colloids

Elasticity index

Concentrated suspension

Concentrated emulsion

Surface softness

\begin{abstract}
A B S T R A C T
We report on the colloidal dynamics and rheology of liquid droplet emulsions and solid particle suspensions over a wide range of volume fractions. Diffusing wave spectroscopy and rheometry were utilized to study the micro/ macroscale properties of 1-eicosene wax particles dispersed in water. By changing the temperature, the wax droplets change their properties from soft to hard or vice versa, which, depending on the density, may affect the system properties in a different way. Our measurements in the high volume fraction regime focus on the elasticity index and the plateau storage modulus, $G_{p}^{\prime}$. The results show a more pronounced increase of the modulus for the wax particle suspensions around the random close packing volume fraction compared to the emulsion.
\end{abstract}

\section{Introduction}

Emulsions are ubiquitous in our daily life and appear in various fields of research and applications such as cosmetics, pharmaceuticals, or food. Due to the large variety of industrial applications where emulsions are employed [1], a plethora of research studies have been carried out to probe the rheological properties of emulsion such as its behavior under shear flow or characterization of the yield stress at high volume fractions [2-4]. Compared to suspensions of hard particles dispersed in a continuum phase, an emulsion is a soft particle system where the liquid-liquid interface is stabilized by surfactant or nanosized particles. Owing to this microscopic difference of the dispersed phase, the macroscopic properties are different in many ways as well. Utilizing the knowledge on the suspensions, it will be useful to compare the characteristics of emulsions that can be physically differentiated from those of suspensions. Thus, the existing models based on suspension systems can be used to describe the micro/macroscopic dynamics of the emulsions as well, such as for example the Krieger-Dougherty equation [5]. While such a comparison is possible for a liquid dispersion it will fail close and above the random close packing or jamming

\footnotetext{
* Corresponding author.

E-mail address: ahnnet@snu.ac.kr (K.H. Ahn).
} 

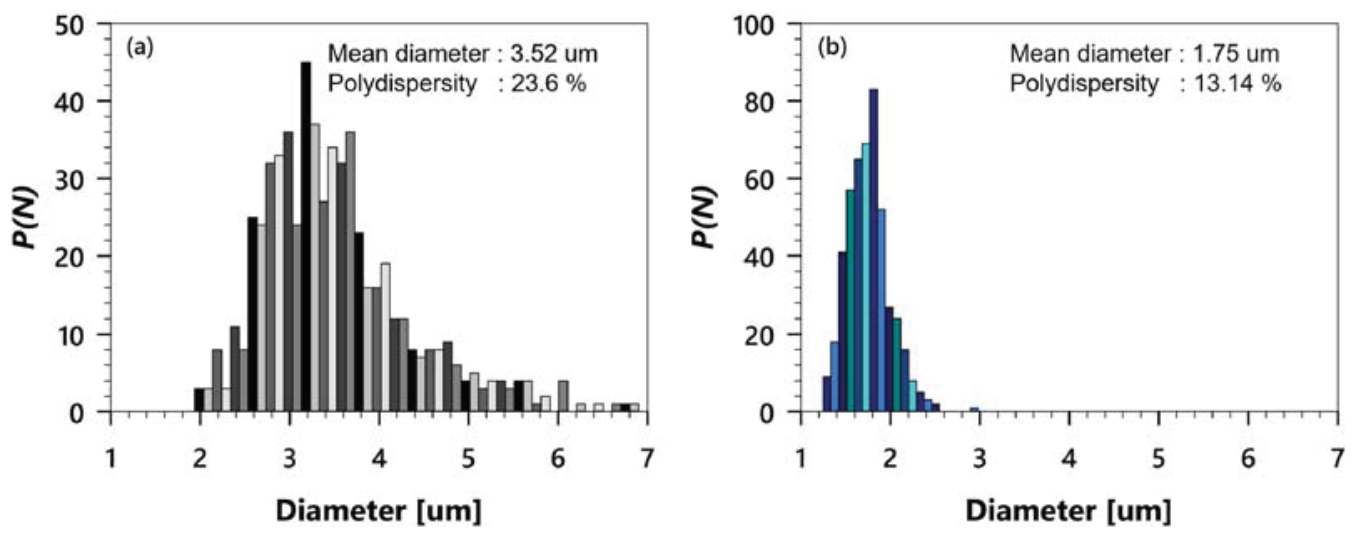

Fig. 1. Number distribution $P(N)$ of the diameter of the emulsion droplets from imaging particle analysis at $30{ }^{\circ} \mathrm{C}$ for sample A (left) and sample B (right). The total number of the particles analyzed is 600 for sample A, and 480 for sample $B$. The values for the mean size $\langle N\rangle$ is given the number weighted average and the polydispersity is the standard deviation of $P$ $(N)$ divided by this mean. transition $\phi=\phi_{r c p}$ when soft particles or droplets are in contact. Emulsions droplets can be compressed and one can access a higher volume fraction regime, well above $\phi_{r c p}$. Emulsion droplets possess soft and adjustable interfaces that deform under high shear or normal stress conditions. The volume fraction regime accessible for hard particle suspensions is normally limited to concentrations below $\phi_{r c p}$ due to its hardness or the vanishing compressibility of the material $[6,2]$. Higher volume fractions can only be reached by breaking or deforming the hard particles irreversibly. Therefore, previous studies at high volume fractions above $\phi>\phi_{r c p}$ addressed intrinsically soft particles such as such as emulsions, polymeric microgels or star polymers and only a few studies compare hard sphere and soft sphere suspensions systems directly [6,2,7-9].To overcome these limitations, and to compare hard and soft particle features using the same material, we study 1-eicosene wax dispersed in water system which has a melting point at $T_{\mathrm{m}}=24.6^{\circ} \mathrm{C}$. This system changes its characteristics from a hard particle suspension to an emulsion or vice versa by changing the temperature. Above $T_{\mathrm{m}}$, wax melts and the particles become soft, while below the melting point, wax solidifies and becomes hard [10]. By changing the temperature, we can dial in softness or hardness while other physical properties of the system such as the solvent composition remain unchanged. We study the rheological properties of the suspensions over a wide range of volume fractions and compare the hard particle suspension and soft particle emulsion system in the liquid state. Moreover, we consider the systems dynamics above the jamming volume fraction at $\phi_{r c p}$ using Diffusing Wave Spectroscopy(DWS) and rheometry. To this end we can access the physical properties of the high volume fraction suspension composed of micron-sized colloids, and can gain insight into the system's internal structure and dynamics. In the experiments we study two different particle or droplet sizes and can thus also test whether the particle size plays an important role.

As explained later in detail, we find, that the system's dynamics and rheology displays distinctly different properties depending on the volume fraction range considered, which we thus separate in three parts. First, at concentrations below 35 vol.\%, denoted as the low volume fraction regime, microstructural changes lead to a characteristic slowing down of the particle or droplet diffusion as described in our earlier work [10]. Macroscopic influences of crowding are manifested by the increase in viscosity. No significant differences between emulsion droplets or hard wax particles were observed. Second, in the intermediate volume fraction regime ranging from 35 vol\% to $\phi_{r c p}$ we find microstructural differences between hard and soft particles, which affects the bulk rheology of the system such as the viscosity or storage modulus of the system. Finally, the third volume fraction regime above $\phi_{r c p}$ is denoted as the high volume fraction regime. In our work, the system can be prepared such that we can reach $\phi>\phi_{r c p}$ not only for the emulsion but even for the suspension, despite the non-deformability of hard particles themselves. This can be realized by using the melting and solidifying process of wax particles, but it also makes the system properties depending on the history of the sample. In this case a high volume fraction emulsion system $\phi>\phi_{r c p}$ is first prepared and the droplets are subsequently solidified by a temperature drop. As a consequence the system becomes a densely packed solid particle suspension (of deformed wax particles).

We study the influence of the softness of particles by studying the bulk rheological properties of both suspensions and emulsion by applying oscillatory shear or a shear flow with a constant shear rate. Our experiments are designed to cover the widest range of volume fractions accessible. In particular we focus on the behavior of suspensions in the high volume fraction regime to clarify the influence of surface elasticity and particle softness by the comparison between emulsion and suspension. The structure of this paper is as follow: in experimental part, the preparation and characterization of the 1-eicosene system and also the experimental methods are described. In the results and discussion part, we first analyze the system for the low and intermediate volume fraction regime and then focus our attention on the high volume fraction regime. At high volume fractions we discuss the elastic properties of both the suspension and the emulsion system. A brief conclusion section will close the paper.

\section{Experimental methods}

We use 1-eicosene in water emulsion stabilized with $10 \mathrm{mM}$ sodium dodecylsulfate (SDS) as a model system. 1-eicosene wax has a melting point at $T_{m}=24.6{ }^{\circ} \mathrm{C}$ and the bulk viscosity in the liquid state at $30{ }^{\circ} \mathrm{C}$ is aprrox. $4 \mathrm{mPa}$ s as measured in house with a rheometer. Samples were prepared using a custom-made couette cell: first, crude emulsions were made by rupturing oil-water-SDS mixture around $3000 \mathrm{rpm}$ over $50{ }^{\circ} \mathrm{C}$ and then the polydispersity of the sample was reduced using the depletion-fractionation method by Bibette [11,12]. Finally, two batches of samples with different size were obtained and the size distribution is plotted in Fig. 1, as derived from a particle sizing and counting analysis of bright-field microscopy images, see also Ref. [10]. Sample A has an mean diameter of $3.52 \mu \mathrm{m}$, polydispersity (standard deviation/mean diameter) of $25.6 \%$ and the volume fractions studied range from 10.7 vol\% to 90.2 vol\%. Meanwhile, sample B has mean diameter of $1.75 \mu \mathrm{m}$, a polydispersity of $13.1 \%$ and the volume fractions studied range from 30.5 vol\% to 82.0 vol\%.

The mass concentration of emulsion droplets is first measured by drying and weighing and then converted to the volume fraction $\phi$ using known density of water and 1-eicosene. The emulsion samples are kept in an oven at $40{ }^{\circ} \mathrm{C}$ and were found to be stable for up to a month but in some cases showed signs of coalescence after 8 weeks and longer. Experiments were done within one month after preparation to ensure that the droplet size distribution in the sample does not vary significantly. Below the melting point $\left(T_{m}=24.6{ }^{\circ} \mathrm{C}\right)$, the system is expected to behave as a particle suspension, because wax particles solidify. However, the transition time to reach full solidification of the 
droplets depends on the final temperature of the sample. At $20^{\circ} \mathrm{C}$, the transition of the emulsion to a suspension takes over $10 \mathrm{~h}$ which we considered too long to prevent drying or aging effects in our experiment. The reason for the relatively slow solidification of the droplets is probably due to the long-lived supercooled liquid state of the wax, which is favoured by the small size of the droplets. However, we have not studied this effect quantitatively. The time for solidification can be reduced to a more convenient time scale by lowering the temperature to $15^{\circ} \mathrm{C}$ or less and at this temperature it takes less then $3 \mathrm{hrs}$ for the system to reach a steady state. Melting takes place much faster already a few degree above $T_{m}$. Thus, above the melting point, the experiments were performed at $30^{\circ} \mathrm{C}$ for the entire range of volume fractions. To make sure that there are no size changes of droplets, due to freezing and melting, we compared the viscosity and storage modulus of the sample over 10 temperature cycles and find no temperature history dependence.

The macroscopic mechanical properties of sample A and B were measured using a Physica MCR300 stress-controlled type rheometer (Anton Paar GmbH, Austria). A solvent trap was used to prevent drying. Before the measurement, for all experiments a preshear at $100 / \mathrm{s}$ for $60 \mathrm{~s}$ and rest for $300 \mathrm{~s}$ is applied to erase the effect of loading. We find that the emulsion (at high temperature) below $\phi_{r c p}$ always shows a Newtonian behavior. For high volume fractions, for both the emulsions and the suspensions, we analyze the rheological behavior under small amplitude oscillatory shear. A strain sweep test is done at a frequency $=1 \mathrm{rad} / \mathrm{s}$ and the strain range of $0.001-100 \%$. Frequency sweep tests are also performed in a range of strain amplitudes where linear viscoelasticity is guaranteed $(0.01-1 \%$ in the volume fraction regime studied).

We also use DWS to analyze the system's internal dynamics under multiple light scattering conditions, owing to high turbidity of the system $[13,14]$. Typical dynamic light scattering or static light scattering methods are not suitable to study such turbid media at concentrations exceeding $10 \mathrm{vol} \%$. In this paper, we obtain a measure of the mean square displacement(MSD) from the commericial DWS Rheolab instrument (LSInstruments AG, Fribourg, Switzerland) in transmission geometry. The path length $L$ of the DWS scattering cell is varied from $0.5 \mathrm{~mm}$ to $5 \mathrm{~mm}$. The accessible range of MSD's is determined by sample's optical transport mean free path $l^{*}$ at a given concentration and the cell path length $L$. From the light scattering experiment, we capture changes of the particle movement as the temperature is varied.

\section{Result and discussions}

For the comparison of the physical properties of emulsion and suspension, we first analyze the behavior of the system at concentrations below $\phi_{r c p}$, i.e. in the low and intermediate volume fraction regime and then above $\phi_{r c p}$ (high volume fraction regime).

\subsection{Low/intermediate volume fraction regime}

We first consider the particle or droplet effective short time self diffusion coefficient $D(\phi)$, derived from the mean square displacements (MSD) probed using DWS: $\left\langle\Delta \vec{r}^{2}(t)\right\rangle=6 D(\phi) t$, as shown in Fig. 2, to evaluate the effect of surface softness on the microscopic behavior of the system. All MSD curves are presented in Fig. S1 for both the suspension and the emulsion system. Diffusion coefficients for the low and intermediate volume fraction regime are calculated from the slope in Fig. S1 for $t>10^{-5} \mathrm{~s}$. We find that the MSD curves vary linearly with time ranging from $10^{-5}$ to $10^{-3} \mathrm{~s}$. To compensate the effect of temperature on the solvent viscosity we plot the dimensionless reduced diffusion coefficient $D(\phi) / D_{0}$ using the diffusion coefficient at infinite dilution as a reference $D_{0}=k T / 6 \pi \eta R$ with $R=1.76 \mu \mathrm{m}$, Fig 1 (a) [6].

At low volume fractions, emulsion and suspension do not show any

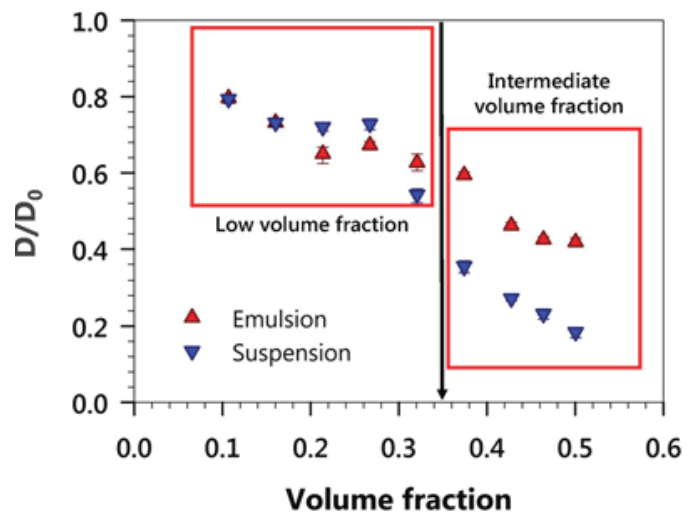

Fig. 2. Reduced diffusion coefficient $D / D_{0}$ for sample A for both the emulsion (red triangles) and the suspensions (blue inverse triangle). The black vertical line indicates $\phi=0.35$ delineating the low volume fraction and the intermediate volume fraction regime in our study. (For interpretation of the references to color in this figure legend, the reader is referred to the web version of this article.)

notable differences in $D$. The situation changes at around $35 \mathrm{vol} \%$, where the data for the hard particle suspension clearly deviates from the soft particle emulsion data. The low/intermediate volume fraction regime is separated according to this criterion at around $35 \mathrm{vol} \%$. In the intermediate volume fraction regime ( $35 \mathrm{vol} \%$ to $\phi_{r c p}$ ), the change 'soft' to 'hard' plays a significant role defining the particles dynamics, although the particles are not yet in contact with each other. For hard particle suspensions, $D(\phi)$ drops dramatically at around $35 \mathrm{vol} \%$, which means the diffusive motion of a single particle begins to be slowed down by the presence of other particles. Such a drop in $D(\phi)$ of the suspension at around $35 \mathrm{vol} \%$ implies the confined diffusive motion of hard particles. This could be a consequence of weak van-der-Waals attractions [15] leading to the formation of aggregation clusters. Strong macroscopic creaming or gravitational settling is not observed on the time scale of the measurements indicating that either the clusters formed are short lived or that some kind of loose particle gel is formed [16].

We obtain information about the motion of individual particles from DWS and we also measure the bulk rheological properties. In Fig 3 , the viscosity of the emulsions is shown for the low to the intermediate volume fraction regime. The emulsion behaves like a Newtonian fluid and we observe no dramatic changes in viscosity at around $35 \mathrm{vol} \%$ which coincides well with the data in Fig. 2 that shows similar trend of

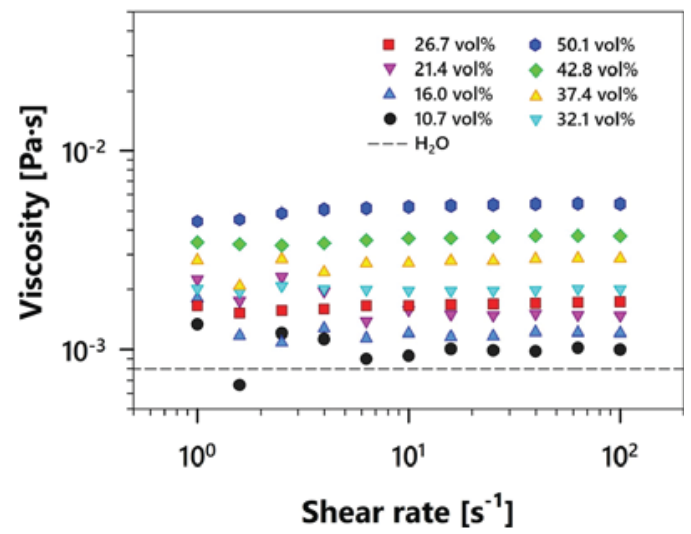

Fig. 3. Symbols show the effective viscosity $\eta(\phi)=\sigma / \dot{\gamma}$ derived from shear flow curves for the emulsion droplets at different volume fractions. $\sigma$ denotes the shear stress. The viscosity $\eta \phi$ displayed in Fig. 4 is extracted from the data over a shear rate $\dot{\gamma}$ ranging from $10 \mathrm{~s}^{-1}$ to $100 \mathrm{~s}^{-1}$ where the data is found to be nearly constant (Newtonian plateau regime). Dashed line: Water viscosity $\eta_{0}=0.8 \mathrm{mPas}$ at $T=30^{\circ} \mathrm{C}$. 


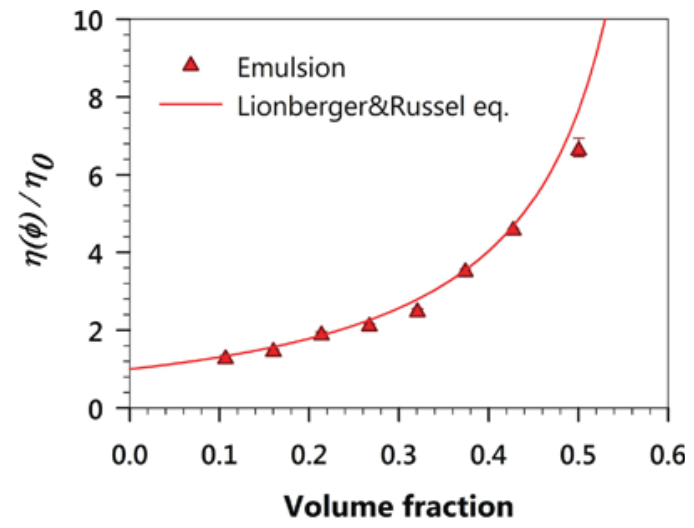

Fig. 4. Reduced viscosity of the emulsion system at $30^{\circ} \mathrm{C}$ as a function of the droplet volume fraction (red triangles) using the water viscosity at $30^{\circ} \mathrm{C}$ as a reference. Solid line: prediction by the semi-empirical expression by Lionberger and Russel [17].

a gradual and continuous decrease for $D / D_{0}(\phi)[2,3]$. In Fig 4 , the reduced viscosity of the emulsion $\eta(\phi) / \eta_{0}$, extracted from the data shown in Fig 3 is plotted. The viscosity of emulsion below $\phi_{r c p}$ fits well with the Lionberger and Russel equation, Eq. (1) [17]. The good agreement between the experimental data and the semi-empirical equation for hard spheres means that the liquid wax emulsion system at high temperatures mimics a hard sphere system up to volume fractions $\phi \leq 50 \%$.

$\eta_{r}=\frac{\eta}{\eta_{0}}=\frac{1+1.5 \phi\left(1+\phi-0.189 \phi^{2}\right)}{1-\phi\left(1+\phi-0.189 \phi^{2}\right)}$

Meanwhile for the suspension, unlike the emulsion, it behaves like a viscoelastic material in which an elastic modulus can be measured for both the low and intermediate volume fraction regime, indicating a system spanning weak network of force chains [18]. Fig. 5 shows the result of the strain sweep test of a $50.05 \mathrm{vol} \%$ sample as an example. Here the storage modulus and loss modulus have plateau values in low strain regime followed by a decrease as strain increases. No local maximum is observed and both $G^{\prime}$ and $G^{\prime}$ decay steadily. The details of the suspension system will be further discussed in next chapter.

\subsection{High volume fraction regime}

Hard particle suspensions cannot be prepared at high volume fractions above $\phi_{r c p}$ due to the non-deformability of particle itself. Here, we use the 1-eicosene wax system to achieve a highly concentrated particle systems. Due to the deformability of the soft particle, the system easily reaches the high volume fraction regime. When particles are solidified

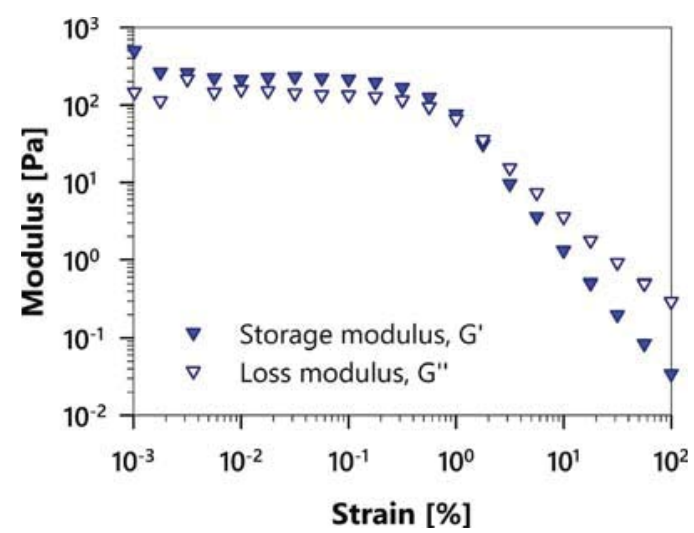

Fig. 5. Strain sweep test at $\omega=1 / s$ for a 50.1 vol $\%$ suspension, sample A ( $G^{\prime}$ : solid symbols, $G^{\prime \prime}$ : open symbols). after the sample composition has been fixed at $\phi / \phi_{r c p}>1$ the hard particle suspension retains a memory of it's prepared state. In this high volume fraction regime, the particles must have a deformed shape with facets at the interface. Images of such facets are clearly visible also for the case of pickering emulsions as shown Ref. [19]. In the limit of very high volume fractions approaching $\phi \rightarrow 1$, the system shows a foam-like structure $[3,2]$ which consists of a network of thin films and plateau borders composed of the continuum phase. The mechanical stability of emulsion system is then determined entirely by the strength of the thin film at the plateau borders [3].

Next, we will have a closer look at the dynamics and the stability of emulsions and suspension in this high concentration regime. For the particulate system, any given particle is at least temporarily surrounded or caged by nearest neighbors. If the concentration is not high enough (i.e. well below $\phi_{r c p}$ ), the movement of particles can be slowed down for a short period of time but the particle can finally escape from its cage by diffusive motion, also called long time diffusion [20]. Meanwhile, as the concentration increases above $\phi_{r c p}$, not only the short time scale diffusive motion but the long time scale diffusive motion (escaping from the cage) of the particles essentially vanishes and particles become permanently trapped in their cages. Initially the motion of particles or droplets in concentrated system is limited to the short time self-diffusion within the cages and long time scale dynamics shows highly correlated dynamics which may be entirely arrested eventually [21,22]. When particles begin to touch the systems jams at $\phi_{r c p}$ and except for a few rattlers the motion of the particles or droplets is determined by their intrinsic softness [23,24]

This microscopic behavior of the particles can be analyzed using the DWS technique which can detect and measure the local positional fluctuations of particles on nanometer length scales, even inside a highly opaque medium. The measured intensity fluctuations of multiply scattered light can be converted to the autocorrelation function and the latter is then used as a measure of the dynamics of the sample. A detailed description of the technique is given for example in ref. [25-27] One can follow the average particle dynamics over wide range of length and time scales. Here we convert the intensity autocorrelation function $g_{2}(\tau)=\langle I(t) I(t+\tau)\rangle_{t} /\left\langle I(t)^{2}\right\rangle_{t}$ of scattered light to the time dependence of the mean square displacement $\left\langle\Delta \vec{r}^{2}(\tau)\right\rangle$, neglecting collective scattering effects $[26,28,29]$.

We quantify the dynamics of the system in different ways depending on the volume fraction regime considered. The viscous properties are defined by the diffusion coefficient obtained from the slope of the MSD, data shown in Fig. 2, for the low and intermediate volume fraction regime. Meanwhile, for the high volume fraction regime, the elastic properties can be quantified by the plateau value of the MSD as shown in Fig. 6.

As the concentration increases, the movement of a single particle over long time scales changes: the diffusion slows down which is reflected as a decrease in slope for the short time MSD, while the MSD reaches a plateau and the plateau value or height is a measure of the solid-like character of the sample due to the restricted motion by other surrounding particles in the long time scale limit. This plateau value reflects the elasticity of the system: as the plateau is lower, the network of force chains becomes tighter and the material is stronger [30]. The elasticity index can be defined, as in denoted in Eq. (2), to quantify the systems' elasticity in a qualitative passive microrheology approach $[31,27,32,33]$.

EI $($ Elasticity index $)=1 /($ plateau height of MSD $)$

Compared to the elastic modulus in bulk rheology, which is observed over the whole range of volume fractions studied, the EI can only be probed in the high volume fraction regime because the particle movement is caged, over the time sales accessible to DWS, only for the volume fraction above $\phi_{r c p}$. In Fig. 7 we show that the volume dependence of the EI is different for the suspension compared to the emulsion: 


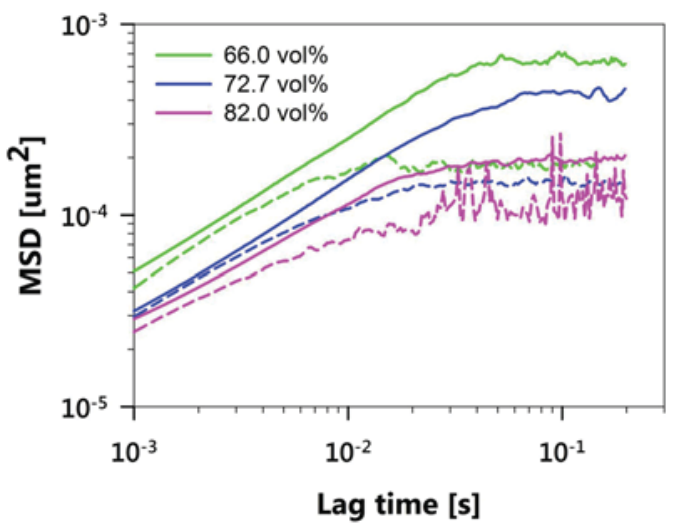

Fig. 6. Mean square displacement curves obtained from the DWS measurements at different volume fractions ranging from $66.0 \mathrm{vol} \%$ to $82.0 \mathrm{vol} \%$ of sample A. The solid line shows the data for the emulsions while the dashed line shows the data for the suspensions.

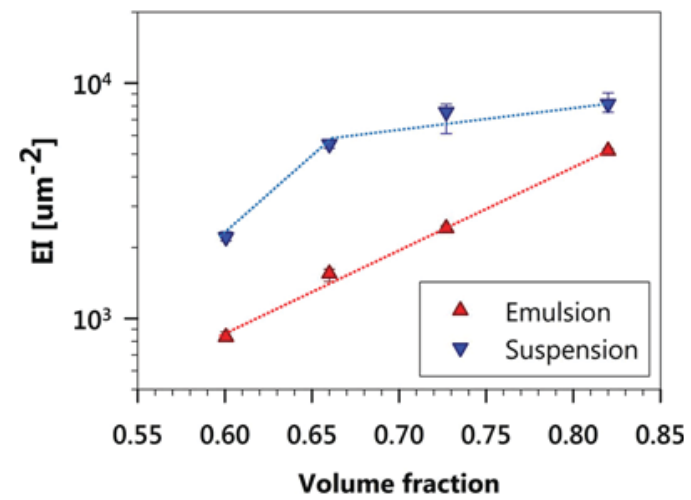

Fig. 7. Elasticity index of the emulsions (red triangles) and suspensions (blue inverted triangles) extracted form the DWS measurements shown in Fig. 6. (For interpretation of the references to color in this figure legend, the reader is referred to the web version of this article.)

for the emulsion the EI continues to increase steadily while for the suspension the EI rapidly jumps to a high value at $\phi \sim \phi_{r c p}$ and then appears increase further only very slowly at higher concentrations. In general the EI of the suspension is substantially higher than that of emulsion over the whole range of volume fractions considered here.

The origin of elasticity of highly concentrated emulsion is different

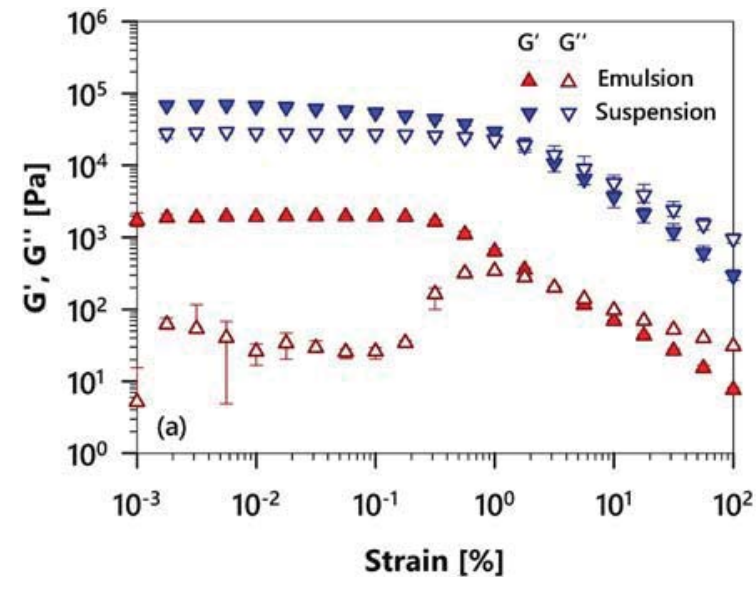

from that of suspension: the elasticity comes from the compressed repulsive droplets permitting the storage of interfacial elastic energy. $[34,35]$ This means the droplets are more and more deformed as the volume fraction increases, and the surface area of the droplets increase, which creates additional storage energy by imposing shear deformation at the interface. The increase of EI for the emulsion shown in Fig 7 can be interpreted in the same way: as the volume fraction increases, more flat facets and contacts between neighboring particles are created, thus the elasticity index increases by the distortion of particle itself. This is due to the deformable characteristics of the soft particle.

However, for the suspension, the situation is very different, even though the particles are also deformed at high volume fraction regime and thus have flat facets at the interfaces. Preformed facets of the hard particles cannot be deformed anymore once the particles are solidified. This implies that the distorted surface areas for single hard particle do not change and therefore the EI of the suspension increases more gently as the shape of the single particle is adjusted to the geometrical constraints at volume fractions above $\phi_{r c p}$, blue symbols in Fig. 7.

To compare the EI to the bulk elastic moduli of the system, we also assessed the macro-rheological properties in high volume fraction regime. Strain sweep and frequency sweep test results are presented as an example for the $87 \mathrm{vol} \%$ of sample B sample (smaller size droplets). In Fig. 8(a), both the emulsion and the suspension show a linear regime until a certain critical strain is reach, known as the yield strain [36]. For the suspension the linear regime is much narrower and we observe no local maximum in loss modulus $G^{\prime \prime}$, while $G^{\prime \prime}$ of the emulsion shows a clear maximum, in agreement with previous studies on model emulsion systems [3]. Interestingly, for the suspension of $87 \mathrm{vol} \%$, the form of the graph is almost the same compared to the data taken in the intermediate volume fraction regime (Fig. 5). The increase in volume fraction merely shifts the modulus upwards by more than two orders of magnitude.

With respect to the frequency dependence of the elastic moduli of the emulsions and the suspension, shown in Fig. 8b, we find that the storage and loss modulus are both are much higher for the suspension than for the emulsion, by a factor of 60 for $G^{\prime}$ and 1800 for $G^{\prime \prime}$ at $\omega=10 \mathrm{rad} / \mathrm{s}$. Moreover the frequency dependency also show some difference: $G^{\prime}$ and $G^{\prime \prime}$ are almost independent of frequency for the emulsion, while for the suspension, $G^{\prime}$ slightly increases with frequency while $G^{\prime \prime}$ remains constant indicating that the system becomes more elastic.

To clearly reveal the difference in the frequency dependence, the storage modulus at frequency $=0.1,1,10,100 \mathrm{rad} / \mathrm{s}$ are plotted versus volume fraction in Fig 9 . Both the suspension and the emulsion show a

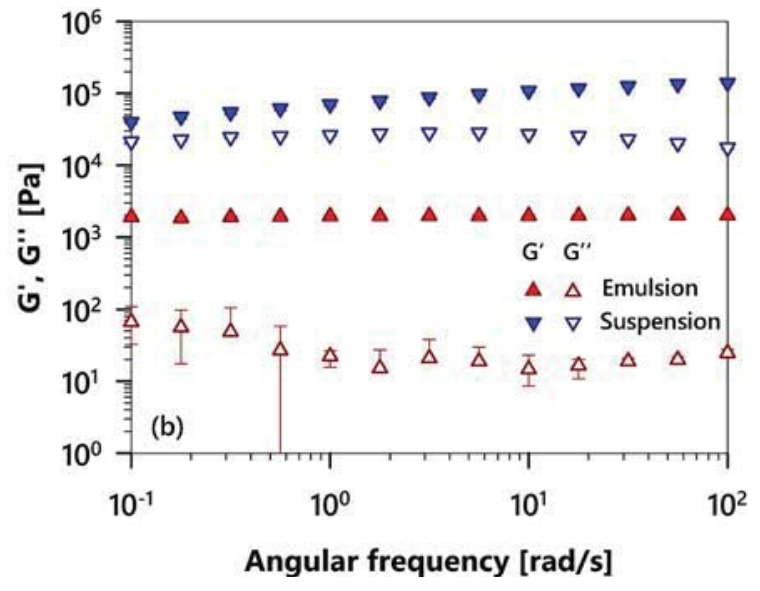

Fig. 8. (a) Dynamic strain sweep for sample B at a volume fraction of $87 \mathrm{vol} \%$. The frequency is set to $1 \mathrm{rad} / \mathrm{s}$ with $G^{\prime}$ denoted by solid symbols and $G^{\prime \prime}$ by open symbols. The data for emulsions is represented by red symbols and suspension data is shown as blue symbols. (b) Dynamic frequency sweep for the same sample with a constant strain of $0.01 \%$ (linear viscoelastic regime). $G^{\prime}$ is denoted by solid and $G^{\prime \prime}$ by open symbols. (For interpretation of the references to color in this figure legend, the reader is referred to the web version of this article.) 

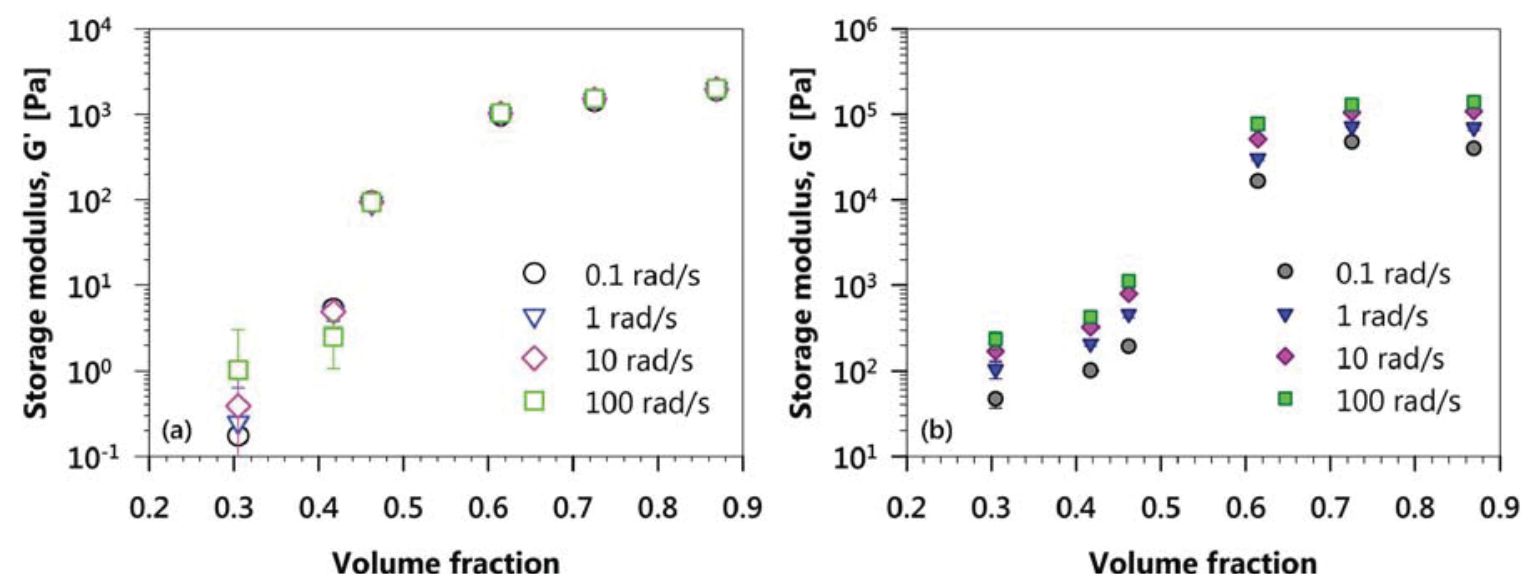

Fig. 9. Storage modulus $G^{\prime}$ from oscillatory shear experiments as a function of volume fraction. Data for different frequencies $\omega=0.1,1,10,100$ rad/s is shown in (a) for the emulsion, at $T=30^{\circ} \mathrm{C}$ and (b) for the suspension, at $T=15^{\circ} \mathrm{C}$.

solid-like response in the high volume fraction regime but follow a rather different frequency dependence. For the emulsions, except for the low volume fraction regime (equal and below $35 \mathrm{vol} \%$ ), little dependence on frequency is observed for $\omega>10 \mathrm{rad} / \mathrm{s}$. Meanwhile the suspension, shows a clear frequency dependence a all volume fractions.

By analogy to the hard sphere system, the elasticity of the emulsion can be represented by a plateau elastic modulus, $G_{p}^{\prime}$. [37] $G_{p}^{\prime}$ is obtained from the analysis of the strain sweep test at frequency $=1 \mathrm{rad} / \mathrm{s}$ in the linear regime for samples A and B with different particle sizes, Fig. 10. Below $\phi_{r c p}$, the droplets of the emulsion are not deformed so the microscopic movement of particles is similar for the emulsion and the suspension [38]. Above $\phi_{r c p}$, the droplets are confined and start to be distorted by the presence of the neighboring particles and this additional deformation results in the elasticity of the emulsion [38]. However, the origin of elasticity of the suspension in this high volume fraction regime is not well understood, since the state can only be reached as long as the wax is liquid and it is not entirely clear microscopically how the particles adapt to this confinement once solidified.

As expected, for the emulsion in the intermediate volume fraction regime, $G_{p}^{\prime}$ is higher when the size is smaller as shown in Fig. S2. However, for the suspension system $G_{p}^{\prime}$ shows no clear size dependence in the high volume fraction regime. To quantitatively analyze this size dependency of $G_{p}^{\prime}$, we introduce Laplace pressure $\propto 1 / R$ to normalize for

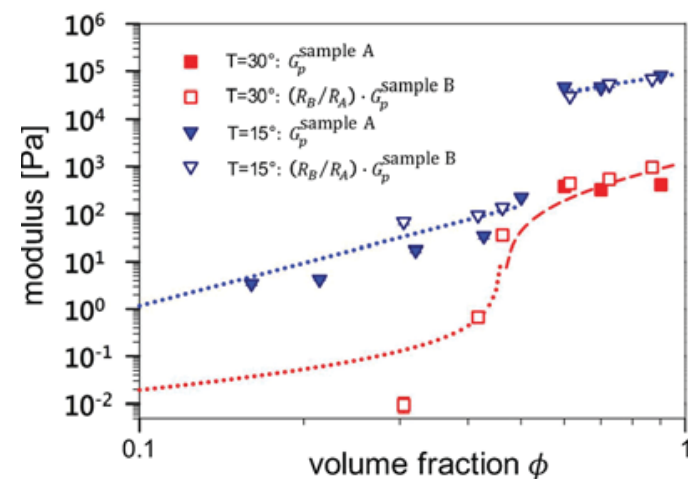

Fig. 10. Plateau storage modulus $G_{p}^{\prime}$ as a function of volume fraction. The results for sample B are rescaled with the size ratio $R_{B} / R_{A} \sim 2$ to take account for the difference in droplet Laplace pressure for the emulsions. Emulsion data is labelled by square symbols and suspension data by inverted triangles. Blue dotted line below $\phi_{r c p}$ indicates a power-law behavior of the suspension [19], $G_{p}^{\prime} \sim \phi^{3}$ while above $\phi_{r c p} G_{p}^{\prime} \sim \phi^{2}$ (blue dotted line). The red dotted line follows the model equation [39] $G_{p}^{\prime} \sim \phi /\left(\phi_{c}-\phi\right)$ for hard spheres which diverges at $\phi_{c} \sim 0.5$, while the red dashed line above $\phi_{r c p}$ is given by the network-spring model of Mason et al. [40] $G_{p}^{\prime} \sim \phi\left(\phi-\phi_{c}\right)$. the size effect in the plateau storage modulus as described in ref. [34]. In our study sample A and B both have the same interfacial tension since the system is based on the same wax(1-eicosene) and surfactant and only the size is different by a factor of about 2, Fig. 1 , so that the Laplace pressure ratio between sample A and B has the value of approximately 2 .

By using Laplace pressure ratio, one can take account of the size effect even without knowing the exact value of the surface tension. In Fig 10 we plot the scaled plateau storage modulus over the whole volume fraction range studied. The data collapse for emulsions and suspensions independently. Surprisingly, the master curve in Fig 10, compensating the droplet size effect just by simple scaling using the Laplace pressure ratio, can be achieved not only for the emulsion but also for the suspension system. The size range explored is very limited however and the experimental uncertainty is almost comparable in some cases, so a firm conclusion about the scaling of the elasticity of the suspension with particle size cannot be derived form our data.

For the emulsion, the size dependence is well adjusted using the Laplace pressure ratio over the whole volume fraction regime as shown in Fig. 10. If we take a closer look at the emulsion case, the $G_{p}^{\prime}$ shows a different concentration dependence in the intermediate and high volume fraction regime compared to the emulsions. For the intermediate volume fraction regime $\left(\phi<\phi_{r c p}\right), G_{p}^{\prime}$ follows the model equation $G_{p}^{\prime} \sim \phi /\left(\phi_{c}-\phi\right)$ with a sharp rise at $\phi_{c} \sim 0.5$ (Fig 10 . red dotted line) [39]. The parameter $\phi_{c}$ denotes a critical volume fraction we find to be approximately $\phi_{c} \sim 0.5$ which is somewhat lower compared to the effective close packing prediction of $\phi_{c} \sim 0.6$ in monodisperse model emulsions [40]. For the high volume fraction regime $\left(\phi>\phi_{r c p}\right), G_{p}^{\prime}$ follows the network-spring model equation $G_{p}^{\prime} \sim \phi\left(\phi-\phi_{c}\right)$ using the same value of $\phi_{c} \sim 0.5$ (Fig 10, red dashed line) [40]. So for the whole volume fraction regime, the 1-eicosene emulsion acts as a fairly standard model emulsion system.

For the suspension case, the size dependence and the concentration dependence of $G_{p}^{\prime}$ changes as the volume fraction increases. For both intermediate and high volume fraction regime, $G_{p}^{\prime}$ shows a power law behavior but the exponent we empirically derive, decreases from 3 to 2 as the concentration increases. Interestingly, we observe a sudden jump of the storage modulus at $\phi \sim 55 \%$ which, as we believe, is a signature of the hard particle nature of the frozen wax particles. Similarly to the emulsions this value is slightly lower than the nominal value of $\phi_{r c p}$. The finite elasticity at higher volume fractions can be understood in terms of the sample preparation protocol and the interaction between the wax particle subject to the forces imposed by the neighbouring particles in contact.

For the concentrated system above $\phi_{r c p}$, the particles should have non-spherical shape for both the emulsion and the suspension [2] and have facets in their interfaces but the characteristics of the facets are 
different. For the emulsion, the droplets are soft and the facets could be modified by the increased osmotic pressure caused by increase of volume fraction or by shear stress. The shape of the particles in the suspension cannot easily be distorted by particle external stresses. The generation of facets in the suspension system could be categorized in two different types: for the low and intermediate volume fraction regime, the facet is created by the crystallization process during the solidification expanding in radial direction from the center of particles, as shown in our previous work [10], meanwhile for high volume fraction regime, the facet is not only generated by crystallization but also induced by the presence of other neighboring particles in the liquid state before the temperature is reduced below $T_{m}$. Moreover during the cooling process reaching $T<T_{m}$ stresses stored and force chains created in the emulsion can become frozen in and the elastic response, i.e. the shear modulus, in the solid wax will then be increased. We can also speculate that the number of contacts imposed by the jammed elastic emulsion [23,24] is retained after solidification. Although particles and droplets in both systems, in the high volume fraction 'overpacking'regime, have facets induced by the presence of other surrounding particles, contacts and facets contribute to the elasticity of the systems in a different way as clearly shown in Fig 7 .

\section{Conclusions}

In this paper, we have studied the micro- and macroscopic behavior of 1-eicosene wax dispersed as micron scale droplets or particles in water over a wide range of volume fractions. Since 1-eicosene has a melting point around $24.6^{\circ} \mathrm{C}$, changing the temperature makes the system alter from an emulsion to a suspension or vice versa without affecting any other physical properties such composition or particle/ droplet size. In previous studies, for the hard particle system, concentrated suspensions above $\phi_{r c p}$ could not be prepared in a well-defined way due to the hard nature of the particles. However, in our work, we could overcome this problem and we could enter the high volume fraction regime for both particulate systems, hard and soft, by just changing the temperature of the wax suspended in system. This way we could broaden the scope of the comparison between emulsions and suspensions covering a wide range of volume fractions. We analyzed the rheological properties and the microscopic dynamic properties using DWS. The results of our experimental study are summarized schematically in the diagram shown in Fig. 11. For a better understanding of the system, we divide the volume fraction axis into 3 parts: the low volume fraction regime (up to $35 \mathrm{vol} \%$ ), the intermediate volume fraction regime (35 vol\% $\sim \phi_{r c p}$ ), and finally the high volume fraction regime (above $\phi_{r c p}$ ) based on the systems microscopic motion and macroscopic flow characteristics.

Around 35 vol\%, which indicates change from the low to the intermediate volume fraction regime, the slope of diffusion coefficient suddenly drops but only for the suspension while it continues to evolve gradually for the emulsion. This implies that the change of the surface softness and the solidification of the particles play a significant role for suspension for the intermediate volume fraction regime likely leading to weak attractive interactions owing to the modified interfacial properties of the wax particles after solidification and a now dominant contribution due to van-der-Waals attraction. At concentrations higher than $\phi \sim \phi_{r c p}$, characterizing the highest possible volume fraction where hard particles are maximally packed or jammed [41],both emulsions and suspensions act as highly elastic, particulate systems but the detailed rheological properties and the microscopic dynamics are very different. For the emulsions, as the volume fraction increases, the droplets deform more and more and the facet area of single particles also increases. This is reflected in the increase of elasticity index (EI) derived from single particle MSD'S, and we can identify the structural disorder and droplet deformation as the origin of sample elasticity. In this situations the modulus $G_{p}^{\prime}$ is droplet size-dependent due to the decrease in Laplace pressure with increase in radius $\propto 1 / R$. However, for the suspension, no additional facet area can be newly created after solidification. The generation of facets is only possible for liquid droplets and the configuration of wax particles is then frozen in during the solidifying process, likely retaining some memory of the number of contacts and force chains created in the emulsion state. The elasticity is then independent of the Laplace pressure of the liquid and only given by the structural conformation and residual elasticity of the solid wax.

\section{Conflicts of interest}

F.S. is a board member and shareholder of LS Instruments AG.

\section{Acknowledgments}

This work was supported from the National Research Foundation of Korea (NRF) grant funded by the Korea Government (MSIT) (No. NRF-

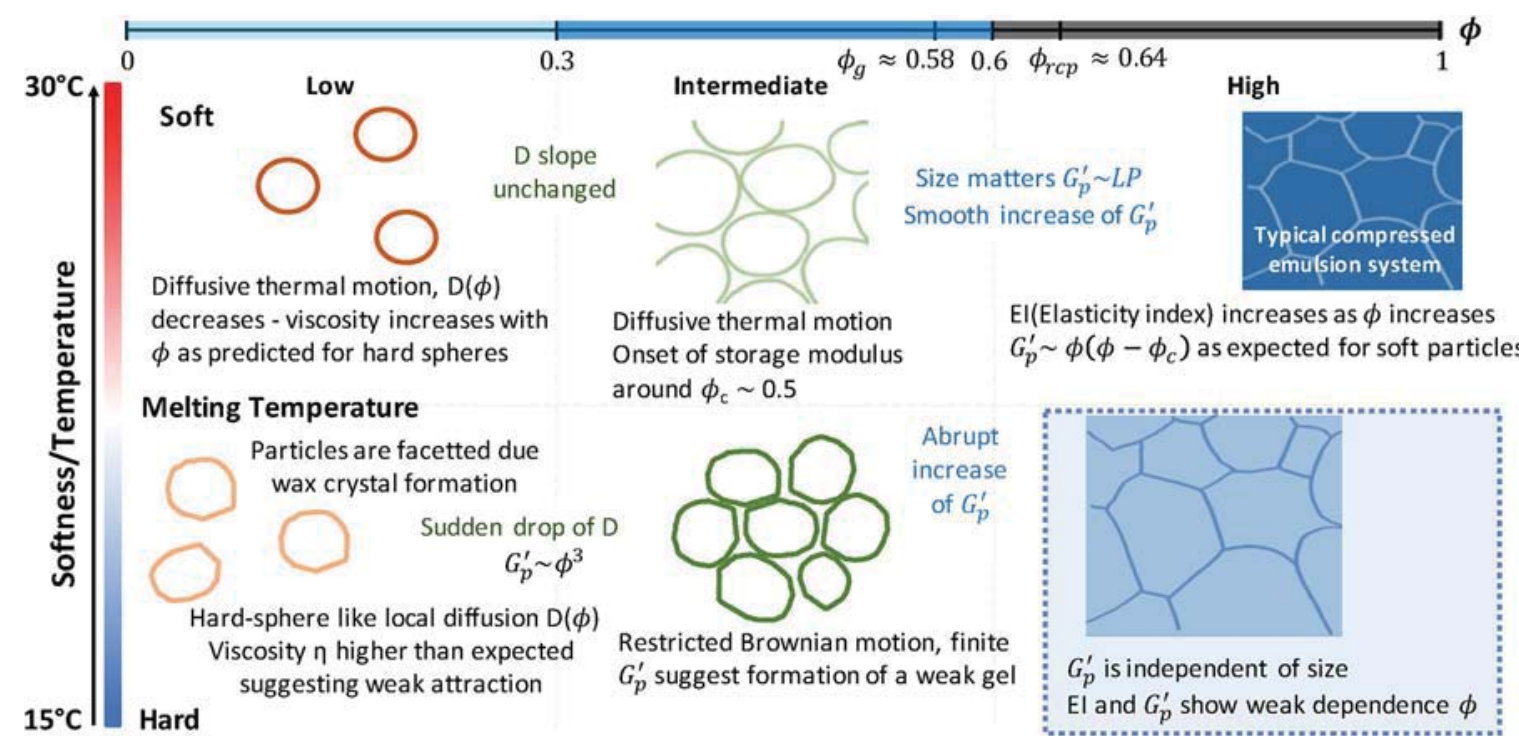

Fig. 11. Schematic representation of the properties of wax emulsions and suspensions in terms of the structural response under shear and microscale diffusive motion for different temperatures and composition. The volume fraction is increasing from left to right and the temperature is increasing from the bottom to the top. 'LP' denotes the Laplace pressure. 
2018R1A5A1024127). F.S. acknowledges funding by the Swiss National Science Foundation through Project No. 169074 and the Adolphe Merkle foundation via the Fribourg Center for Nanomaterials (FriMat). This work benefitted from support of the Swiss National Science Foundation through the National Center of Competence in Research (NCCR) Bio-Inspired Materials.

\section{Appendix A. Supplementary data}

Supplementary data associated with this article can be found, in the online version

\section{References}

[1] S. Tajik, B. Nasernejad, A. Rashidi, Surface modification of silica-graphene nanohybrid as a novel stabilizer for oil-water emulsion, Korean J. Chem. Eng. 34 (9) (2017) 2488-2497, https://doi.org/10.1007/s11814-017-0125-y.

[2] T.G. Mason, J.N. Wilking, K. Meleson, C.B. Chang, S.M. Graves, Nanoemulsions: formation, structure, and physical properties, J. Phys.: Condens. Matter 18 (2006) 635-666, https://doi.org/10.1088/0953-8984/18/41/R01.

[3] T.G. Mason, J. Bibette, D.A. Weitz, Yielding and flow of monodisperse emulsions, J. Colloid Interface Sci. 179 (2) (1996) 439-448, https://doi.org/10.1006/jcis.1996. 0235.

[4] T.G. Mason, J. Bibette, Emulsification in Viscoelastic Media, Phys. Rev. Lett. 77 (16) (1996) 3481-3484, https://doi.org/10.1103/PhysRevLett.77.3481.

[5] H.A. Barnes, Rheology of emulsions - a review, Colloids Surf. A: Physicochem. Eng. Aspects (1994), https://doi.org/10.1016/0927-7757(93)02719-U.

[6] M. Alexander, L.F. Rojas-Ochoa, M. Leser, P. Schurtenberger, Structure, dynamics, and optical properties of concentrated milk suspensions: an analogy to hard-sphere liquids, J. Colloid Interface Sci. 253 (1) (2002) 35-46, https://doi.org/10.1006/ jcis.2002.8452.

[7] N. Koumakis, A. Pamvouxoglou, A. Poulos, G. Petekidis, Direct comparison of the rheology of model hard and soft particle glasses, Soft Matter 8 (15) (2012) 4271-4284.

[8] F. Scheffold, P. Díaz-Leyva, M. Reufer, N.B. Braham, I. Lynch, J.L. Harden, Brushlike interactions between thermoresponsive microgel particles, Phys. Rev. Lett. 104 (12) (2010) 128304.

[9] C. Likos, H. Löwen, M. Watzlawek, B. Abbas, O. Jucknischke, J. Allgaier, D. Richter, Star polymers viewed as ultrasoft colloidal particles, Phys. Rev. Lett. 80 (20) (1998) 4450 .

[10] J. Yoon, F. Cardinaux, C. Lapointe, C. Zhang, T.G. Mason, K.H. Ahn, F. Scheffold, Brownian dynamics of colloidal microspheres with tunable elastic properties from soft to hard, Colloids Surf. A: Physicochem. Eng. Aspects 546 (2018) 360-365, https://doi.org/10.1016/j.colsurfa.2018.02.046.

[11] T.G. Mason, Osmotically driven shape-dependent colloidal separations, Phys. Rev. E 66 (6) (2002) 4, https://doi.org/10.1103/PhysRevE.66.060402.

[12] J. Bibette, Depletion interactions and fractionated crystallization for polydisperse emulsion purification, J. Colloid Interface Sci. 147 (2) (1991) 474-478.

[13] G. Maret, P. Wolf, Multiple light scattering from disordered media. the effect of Brownian motion of scatterers, Zeitschrift für Physik B Condensed Matter 65 (4) (1987) 409-413.

[14] D.J. Pine, D.A. Weitz, P.M. Chaikin, E. Herbolzheimer, Diffusing wave spectroscopy, Phys. Rev. Lett. 60 (12) (1988) 1134-1137, https://doi.org/10.1103/PhysRevLett. 60.1134 .

[15] J.N. Israelachvili, Intermolecular and Surface Forces, Academic Press, 2011.

[16] E. Zaccarelli, Colloidal gels: equilibrium and non-equilibrium routes, J. Phys.: Condens. Matter 19 (32) (2007) 323101.
[17] A.J. Banchio, G. Nägele, Short-time transport properties in dense suspensions: from neutral to charge-stabilized colloidal spheres, J. Chem. Phys. 128 (10) (2008) 104903, https://doi.org/10.1063/1.2868773.

[18] V. Trappe, V. Prasad, L. Cipelletti, P. Segre, D.A. Weitz, Jamming phase diagram for attractive particles, Nature 411 (6839) (2001) 772.

[19] M.N. Lee, H.K. Chan, A. Mohraz, Characteristics of pickering emulsion gels formed by droplet bridging, Langmuir 28 (6) (2012) 3085-3091, https://doi.org/10.1021/ la203384f.

[20] G. Nägele, On the dynamics and structure of charge-stabilized suspensions, Phys. Rep. 272 (5-6) (1996) 215-372.

[21] G.L. Hunter, E.R. Weeks, The physics of the colloidal glass transition, Rep. Prog. Phys. 75 (6) (2012) 066501.

[22] J. Bergenholtz, W.C.K. Poon, M. Fuchs, Gelation in model colloid - polymer mixtures, Langmuir 19 (10) (2003) 4493-4503.

[23] M. van Hecke, Jamming of soft particles: geometry, mechanics, scaling and isostaticity, J. Phys.: Condens. Matter 22 (3) (2009) 033101.

[24] A.J. Liu, S.R. Nagel, The jamming transition and the marginally jammed solid, Annu. Rev. Condens. Matter Phys. 1 (1) (2010) 347-369.

[25] D. Weitz, D. Pine, Diffusing-wave spectroscopy, Dynamic Light Scattering: The Method and some Applications, Oxford University Press, 1993, pp. 652-720.

[26] F. Scheffold, P. Schurtenberger, Light scattering probes of viscoelastic fluids and solids, Soft Mater. 1 (2) (2003) 139-165, https://doi.org/10.1081/SMTS120022461 URL http://www.informaworld.com/smpp/titlecontent $=$ t713597297.

[27] E.M. Furst, T.M. Squires, Microrheology, Oxford University Press, 2017.

[28] C. Zhang, M. Reufer, D. Gaudino, F. Scheffold, Improved diffusing wave spectroscopy based on the automatized determination of the optical transport and absorption mean free path, Korea-Australia Rheol. J. 29 (4) (2017) 241-247.

[29] H.S. Kim, N. Senbil, C. Zhang, F. Scheffold, T.G. Mason, Diffusing wave microrheology of highly scattering concentrated monodisperse emulsions, Proc. Natl. Acad. Sci. (2019) 201817029, https://doi.org/10.1073/pnas.1817029116.

[30] S. Romer, H. Bissig, P. Schurtenberger, F. Scheffold, Rheology and internal dynamics of colloidal gels from the dilute to the concentrated regime, Europhys. Lett. 108 (4) (2014) 48006.

[31] C. Tisserand, M. Fleury, L. Brunel, P. Bru, G. Meunier, Passive microrheology for measurement of the concentrated dispersions stability, UK Colloids 2011, Springer Berlin Heidelberg, Berlin, Heidelberg, 2012, pp. 101-105, https://doi.org/10. 1007/978-3-642-28974-3_17.

[32] T.A. Waigh, Microrheology of complex fluids, Rep. Prog. Phys. 68 (3) (2005) 685.

[33] T.A. Waigh, Advances in the microrheology of complex fluids, Rep. Prog. Phys. 79 (7) (2016) 074601.

[34] T. Mason, M.-D. Lacasse, G. Grest, D. Levine, J. Bibette, D. Weitz, Osmotic pressure and viscoelastic shear moduli of concentrated emulsions, Phys. Rev. E 56 (3) (1997) 3150-3166, https://doi.org/10.1103/PhysRevE.56.3150.

[35] F. Cardinaux, T.G. Mason, F. Scheffold, Elasticity and glassy dynamics of dense emulsions, AIP Conf. Proceed. 1518 (2013) 222-226, https://doi.org/10.1063/1. 4794571.

[36] D. Bonn, M.M. Denn, L. Berthier, T. Divoux, S. Manneville, Yield stress materials in soft condensed matter, Rev. Modern Phys. 89 (3) (2017) 035005.

[37] T. Mason, New fundamental concepts in emulsion rheology, Curr. Opin. Colloid Interface Sci. 4 (3) (1999) 231-238, https://doi.org/10.1016/S1359-0294(99) 00035-7.

[38] D.R.P. Pascal, A.A. Schweitzer, F. Pessac, T.G. Mason, Elastic. Compressed Emulsions 75 (10) (1995) 2051-2054.

[39] S.S. Datta, D.D. Gerrard, T.S. Rhodes, T.G. Mason, D.A. Weitz, Rheology of Attractive Emulsions, Phys. Rev. E (2011), https://doi.org/10.1103/PhysRevE.84 041404.

[40] T.G. Mason, J. Bibette, D.A. Weitz, Elasticity of compressed emulsions, Phys. Rev. Lett. 75 (10) (1995) 2051-2054, https://doi.org/10.1103/PhysRevLett.75.2051.

[41] S. Torquato, T.M. Truskett, P.G. Debenedetti, Is random close packing of spheres well defined? Phys. Rev. Lett. 84 (10) (2000) 2064. 